\title{
Expression for the transport of ions through a spherical ion-exchange membrane
}

\author{
Jyh-Ping Hsu*, Ji-Ming Jiang \\ Department of Chemical Engineering, National Taiwan University, Taipei 10617, Taiwan \\ Received 24 July 1998; received in revised form 24 July 1998; accepted 7 January 1999
}

\begin{abstract}
The current efficiency of a spherical ion-selective membrane is examined theoretically. A perturbation method is adopted to solve the Nernst-Planck equation governing the spatial variation of the concentration of ions in the membrane phase for the case where the distribution of fixed charge is a linear function of radial position. The result of numerical simulation reveals that the qualitative behavior of spherical membranes is similar to that of cylindrical membranes. For example, if the fixed charge is uniformly distributed, the current efficiency is independent of both the inner radius (or curvature) of a membrane and the applied current density, and the current efficiency of a membrane can be raised by choosing its parameters appropriately. A general expression for planar, cylindrical, and spherical membranes is also discussed. (C) 1999 Elsevier Science B.V. All rights reserved.
\end{abstract}

Keywords: Spherical ion-exchange membrane; Current efficiency; Nernst-Planck equation; Perturbation solution; Linear fixed charge distribution

\section{Introduction}

The performance of an ion-selective membrane depends on its properties and the associated operating conditions. The former includes, for example, the geometry and the distribution of functional groups, and the latter includes the flow field of the system under consideration, the applied current density, and the arrangement of membranes in a stack. The distribution of functional groups in a membrane is directly related to the spatial variation of the fixed charge, which is found to correlate with its current

\footnotetext{
*Corresponding author. Fax: +886-2-236-23040; e-mail: t8504009@ccms.ntu.edu.tw
}

efficiency. Reiss and Bassignana [1], for instance, showed that the current efficiency of a membrane with a sandwich-type fixed charge distribution is higher than that of a membrane with a uniformly distributed fixed charge distribution. On the other hand, Manzanares et al. [2] pointed out that raising the current efficiency of a membrane by nonuniformly distributing the fixed charge it carries cannot be guaranteed. The relation between the fixed charge distribution and the current efficiency was examined by Manzanares et al. [3], and the analysis extended by Hsu and Ting [4] in a recent study. An intuitive parameter was proposed in the latter to measure the degree of uniformity of a fixed charge distribution, and its relation with current efficiency summarized. 
The transport of ions is described by the NernstPlanck equation. Strictly speaking, it is applicable to a membrane having a uniform property. Under idealized conditions, such as the concentration of solution being low and the fluid velocity in the membrane phase negligible, it is also applicable to a more general type of membrane [5]. This is because the cross product terms in a Nernst-Planck equation can be neglected. In this case, the transport of an ion specie is related to its concentration and the applied electric field only. The simplified Nernst-Planck equation considers two types of driving force for ion transport: concentration gradient and electric-potential gradient. To determine the latter, a Poisson equation, which describes the spatial variation of the electrical potential, must be considered. In other words, a set of coupled NernstPlanck and Poisson equations needs to be solved simultaneously. In general, solving these equations analytically is nontrivial, and choosing a numerical scheme is inevitable. The problem under consideration, however, can be simplified by assuming local electroneutrality. In this case, the Poisson equation becomes a Laplace equation, which can be solved easily. It was shown that assuming local electroneutrality is appropriate if the concentration of fixed charge in a membrane is sufficiently high [6].

Although reported results concerning the performance of an ion-selective membrane are abundant in the literature, most of them are limited to planar geometry for a simpler mathematical treatment and experimental design. Often, it is assumed that there is no lateral flow, that is, the transport of ions is in a direction perpendicular to the membrane surface [7] and the effect of concentration polarization is neglected. A hollow-fiber type of device was proposed by Hsu and Yang [8] recently. The device has a circular cross section which is capable of providing a large (surface area/volume) ratio, and, therefore, the transport of ion can be conducted in a more efficient manner than a planar membrane. The analysis was also extended by Hsu and Liu [9] to an annular membrane having an elliptic cross section. In the present study, the behavior of a spherical membrane is examined. Compared with a cylindrical membrane, it is capable of providing an even larger (surface area/volume) ratio. A perturbation method is proposed for the resolution of the governing equation.

\section{Analysis}

By referring to Fig. 1, we consider a spherical membrane of width $W$. Let $r$ be the position variable, and $r_{\mathrm{o}}$ and $r_{\mathrm{i}}$ the outer and inner radii of the membrane, respectively. The origin of the coordinate is located at the center of the membrane. Let $C_{\mathrm{i}}$ and $C_{\mathrm{o}}$ be the bulk concentrations of ions inside and outside the membrane. $J_{1}$ and $J_{2}$ denote, respectively, the fluxes of cations and anions, and $I$ the applied current. For a simpler mathematical treatment, the effect of the boundary layers near liquid-membrane interfaces on the performance of the membrane is neglected. The transport of ions in a steady state is governed by the Nernst-Planck equation [2]. Assuming 1: 1 electrolyte solution, we have

$$
\frac{J_{j}}{r^{2}}=-D_{j}\left[\frac{\mathrm{d} C_{j}}{\mathrm{~d} r}+(-1)^{j+1} \frac{q}{k_{\mathrm{B}} T} \frac{\mathrm{d} \phi}{\mathrm{d} r} C_{j}\right], \quad j=1,2
$$

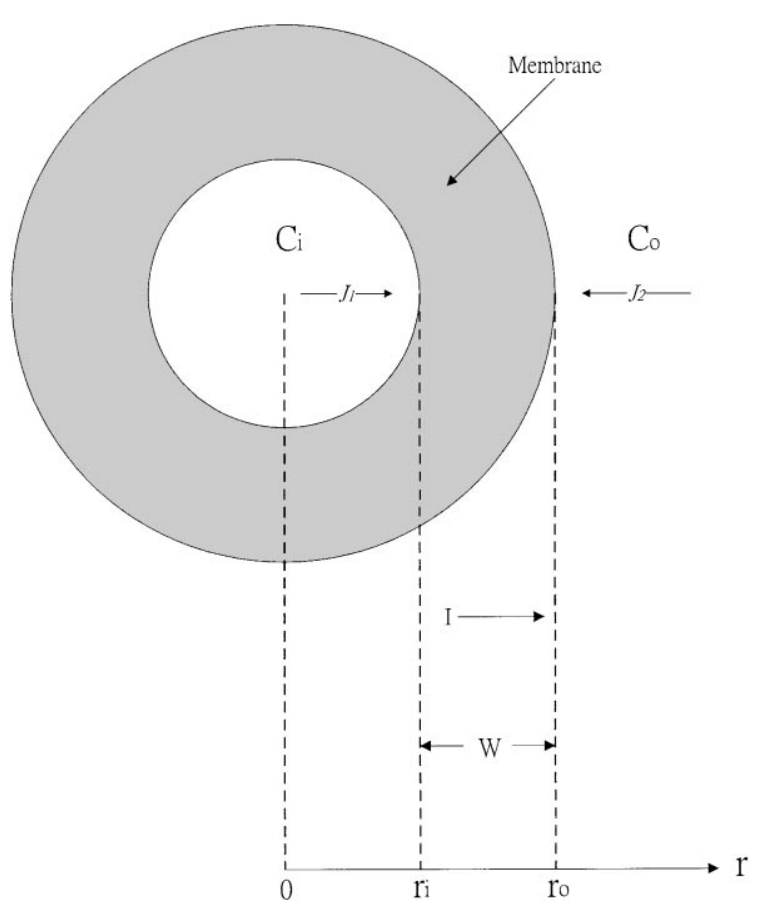

Fig. 1. Schematic representation of the system under consideration. $W$ denotes the width of a membrane, $r$ the position variable, $r_{\mathrm{o}}$ and $r_{\mathrm{i}}$, respectively, the outer and inner radii, $C_{\mathrm{i}}$ and $C_{\mathrm{o}}$ the bulk concentrations of ions inside and outside the membrane, respectively, $J_{1}$ and $J_{2}$, respectively, the fluxes of cation and anion, and $I$ the applied current. 
where $D_{j}$, and $C_{j}$ are, respectively, the diffusivity and the concentration of ion species $j$ ( $j=1$ for cations, $j=2$ for anions), $q$ the elementary charge, $\phi$ the electrical potential, and $k_{\mathrm{B}}$ and $T$ the Boltzmann constant and the absolute temperature, respectively. Note that the flux density of ion species $j,\left(J_{j} / r^{2}\right)$, is independent of the position in a membrane at the steady state. Suppose that $D_{1}=D_{2}=D$. The electrical potential is governed by the following Poisson equation [10]

$\frac{\mathrm{d}^{2} \phi}{\mathrm{d} r^{2}}+\frac{2}{r} \frac{\mathrm{d} \phi}{\mathrm{d} r}=-\frac{4 \pi q}{\omega}\left(C_{1}-C_{2}-C_{3}\right)$

where $\omega$ denotes the dielectric constant, and $C_{3}$ the concentration of fixed charge in the membrane. Without loss of generality, we assume that the fixed charge is negative.

The current $I$ can be expressed as

$$
I=F\left(J_{1}-J_{2}\right)
$$

where $F$ is the Faraday constant. Note that, in the present case, $J_{1}$ is positive and $J_{2}$ negative. Eqs. (1)(3) can be rewritten in the following scaled forms:

$$
\begin{aligned}
& -\frac{\mathrm{d} p}{\mathrm{~d} \xi}-p \frac{\mathrm{d} \psi}{\mathrm{d} \xi}=\frac{h_{\mathrm{c}}}{\xi^{2}} \\
& -\frac{\mathrm{d} n}{\mathrm{~d} \xi}+n \frac{\mathrm{d} \psi}{\mathrm{d} \xi}=\frac{g_{\mathrm{c}}}{\xi^{2}} \\
& \frac{\mathrm{d}^{2} \psi}{\mathrm{d} \xi^{2}}+\frac{2}{\xi} \frac{\mathrm{d} \psi}{\mathrm{d} \xi}=-L^{2}[p-n-\rho(\xi)] \\
& i=h_{\mathrm{c}}-g_{\mathrm{c}}
\end{aligned}
$$

In these expression, $\quad \psi=q \phi / k_{\mathrm{B}} T, \quad p=C_{1} / C_{\mathrm{i}}$, $n=\quad C_{2} / C_{\mathrm{i}}, \quad \rho(\xi)=C_{3} / C_{\mathrm{i}}, \quad \xi=r / W, \quad h_{\mathrm{c}}=h r_{\mathrm{i}} / W$, $g_{\mathrm{c}}=g r_{\mathrm{i}} / W$, and

$$
\begin{aligned}
h & =\frac{J_{1}}{D C_{\mathrm{i}} r_{\mathrm{i}}} \\
g & =\frac{J_{2}}{D C_{\mathrm{i}} r_{\mathrm{i}}} \\
L & =W\left(\frac{4 \pi q^{2} C_{\mathrm{i}}}{\omega k_{\mathrm{B}} T}\right)^{1 / 2} \\
i & =h_{\mathrm{c}}-g_{\mathrm{c}}=\frac{I}{D C_{\mathrm{i}} F W}
\end{aligned}
$$

If the concentration of fixed charge is high, local electroneutrality can be assumed, then the right-hand side of Eq. (6) vanishes, that is,

$p-n-\rho(\xi)=0$

Therefore, if $p$ is known, $n$ can be evaluated from this expression.

The current efficiency, $\eta$, is defined as [3]

$\eta=\frac{|h|}{|h|+|g|}$

According to this definition, $0.5 \leq \eta \leq 1$. If $\eta=0.5$, half of the current is carried by cations, and the other half by anions. In this case, the membrane is useless as far as the ion separation is concerned. On the other hand, if $\eta=1$, the transport of anion is totally blocked by the membrane. Thus, the performance of a membrane can be measured by $\eta$; the higher its value, the better the performance.

For an illustration, we consider a general linear fixed charge distribution

$\rho(\xi)=\alpha+\beta \xi$

We define the scaled mean fixed charge concentration, $\gamma$, as

$$
\begin{aligned}
\gamma & =\left[4 \pi \int_{\xi_{\mathrm{i}}}^{\xi_{\mathrm{o}}}(\alpha+\beta \xi) \xi^{2} \mathrm{~d} \xi\right] /\left[\frac{4 \pi}{3}\left(\xi_{\mathrm{o}}^{3}-\xi_{\mathrm{i}}^{3}\right)\right] \\
& =\left[\frac{\alpha}{3}\left(\xi_{\mathrm{o}}^{3}-\xi_{\mathrm{i}}^{3}\right)+\frac{\beta}{4}\left(\xi_{\mathrm{o}}^{4}-\xi_{\mathrm{i}}^{4}\right)\right] /\left[\frac{1}{3}\left(\xi_{\mathrm{o}}^{3}-\xi_{\mathrm{i}}^{3}\right)\right]
\end{aligned}
$$

where $\xi_{\mathrm{i}}=r_{\mathrm{i}} / \mathrm{W}$, and $\xi_{\mathrm{o}}=r_{\mathrm{o}} / W$.

On the basis of Eqs. (4), (5), (8) and (10), we have

$$
\frac{\mathrm{d} \psi}{\mathrm{d} \xi}=\frac{g_{\mathrm{c}}-h_{\mathrm{c}}-\beta \xi^{2}}{\xi^{2}(2 p-\alpha-\beta \xi)}
$$

Eqs. (4) and (12) lead to

$$
\frac{\mathrm{d} p}{\mathrm{~d} \xi}=\frac{p\left(h_{\mathrm{c}}+g_{\mathrm{c}}-\beta \xi^{2}\right)-h_{\mathrm{c}}(\alpha+\beta \xi)}{\xi^{2}(\alpha+\beta \xi-2 p)}
$$

The boundary conditions associated with this equation are

$$
\begin{aligned}
& p=p^{\mathrm{i}}, \xi=\xi_{\mathrm{i}} \\
& p=p^{\mathrm{o}}, \xi=\xi_{\mathrm{o}}
\end{aligned}
$$


where $p^{\mathrm{i}}$ and $p^{\mathrm{o}}$ are, respectively, the scaled concentrations of cations at the inner and outer boundaries of a membrane. Suppose that Donnan equilibrium is established at the liquid-membrane interface. Then, it can be shown that $p^{\mathrm{i}}$ and $p^{\mathrm{o}}$ are related by [1]

$p^{\mathrm{i}}=\frac{1}{2} \rho\left(\xi_{\mathrm{i}}\right)+\left[\frac{1}{4} \rho^{2}\left(\xi_{\mathrm{i}}\right)+1\right]^{1 / 2}$

$p^{\mathrm{o}}=\frac{1}{2} \rho\left(\xi_{\mathrm{o}}\right)+\left[\frac{1}{4} \rho^{2}\left(\xi_{\mathrm{o}}\right)+\theta^{2}\right]^{1 / 2}$

where $\theta=C_{\mathrm{o}} / C_{\mathrm{i}}$. For simplicity, we assume that $\theta=1$ in the following discussion. Note that Eq. (13) contains two unknowns, $h$ and $g$, and we have one more boundary condition to consider, Eq. (13b). The current efficiency can be determined through a trial-anderror procedure [8]. For a given current density, an $\eta$ is assumed, and $h$ and $g$ are evaluated by Eqs. (7a) and (7b). The corresponding boundary conditions are determined. Based on these conditions, a new $\eta$ is calculated. These steps are repeated until a convergent result is obtained.

Suppose that the width of the membrane is much thinner than its inner radius, that is, $\left(\xi_{\mathrm{o}}-\xi_{\mathrm{i}}\right) \ll \xi_{\mathrm{i}}$. In
Substituting this expression into Eq. (13), we obtain

$$
\begin{aligned}
& \frac{\mathrm{d}}{\mathrm{d} \xi}\left(\sum_{j=0}^{\infty} p_{j} \varepsilon^{j}\right) \\
& =\frac{\left(\sum_{j=0}^{\infty} p_{j} \varepsilon^{j}\right)\left\{h_{\mathrm{c}}+g_{\mathrm{c}}-\beta \xi_{\mathrm{i}}^{2}\left[1+\varepsilon\left(\xi-\xi_{\mathrm{i}}\right)^{2}\right]\right\}-h_{\mathrm{c}}(\alpha+\beta \xi)}{\xi_{\mathrm{i}}^{2}\left[1+\varepsilon\left(\xi-\xi_{\mathrm{i}}\right)\right]^{2}\left(\alpha+\beta \xi-2 \sum_{j=0}^{\infty} p_{j} \xi^{j}\right)} \\
& \cong\left\{\left[p_{0}\left(h+g-\beta \xi_{\mathrm{i}}\right)-h(\alpha+\beta \xi)\right]+\varepsilon\left[p_{1}\left(h+g-\beta \xi_{i}\right)\right]\right. \\
& \left.-2 p_{0} \beta \xi_{\mathrm{i}}\left(\xi-\xi_{\mathrm{i}}\right)\right]+\varepsilon^{2}\left[p_{2}\left(h+g-\beta \xi_{\mathrm{i}}\right)-2 p_{1} \beta \xi_{\mathrm{i}}\left(\xi-\xi_{\mathrm{i}}\right)\right] \\
& \left.-p_{0} \beta \xi_{\mathrm{i}}\left(\xi-\xi_{\mathrm{i}}\right)^{2}\right\} /\left\{\xi _ { \mathrm { i } } \left\{\left(\alpha+\beta-2 p_{0}\right)+\varepsilon\left[2\left(\alpha+\beta \xi-2 p_{0}\right)\left(\xi-\xi_{\mathrm{i}}\right)\right.\right.\right. \\
& \left.\left.\left.-2 p_{1}\right]+\varepsilon^{2}\left[-4 p_{1}\left(\xi-\xi_{\mathrm{i}}\right)-2 p_{2}+\left(\xi-\xi_{\mathrm{i}}\right)^{2}\left(\alpha+\beta \xi-2 p_{0}\right)\right]\right\}\right\}
\end{aligned}
$$

The expression $\left[1 /\left(x+\varepsilon y+\varepsilon^{2} z\right)\right]$ can be expanded in terms of $\varepsilon$ as [8]

$\frac{1}{x+\varepsilon y+\varepsilon^{2} z}=\frac{1}{x}-\frac{y}{x} \varepsilon+\frac{y^{2}-x z}{x^{3}} \varepsilon^{2}+\cdots$

Expanding Eq. (17) based on this expression, and collecting terms of the same order of $\varepsilon$, we have for $\varepsilon^{0}$

$$
\frac{\mathrm{d} p_{0}}{\mathrm{~d} \xi}=\frac{p_{0}\left(h+g-\beta \xi_{\mathrm{i}}\right)-h(\alpha+\beta \xi)}{\xi_{\mathrm{i}}\left(\alpha+\beta \xi-2 p_{0}\right)}
$$

for $\varepsilon^{1}$

$\frac{\mathrm{d} p_{1}}{\mathrm{~d} \xi}=\frac{p_{1}\left(h+g-\beta \xi_{\mathrm{i}}\right)-2 p_{0} \beta \xi_{\mathrm{i}}\left(\xi-\xi_{\mathrm{i}}\right)}{\xi_{\mathrm{i}}\left(\alpha+\beta \xi-2 p_{0}\right)}-\frac{\left[-2 p_{1}+2\left(\xi-\xi_{\mathrm{i}}\right)\left(\alpha+\beta \xi-2 p_{0}\right)\right]\left[p_{0}\left(h+g-\beta \xi_{\mathrm{i}}\right)-h(\alpha+\beta \xi)\right]}{\xi_{\mathrm{i}}\left(\alpha+\beta \xi-2 p_{0}\right)^{2}}$

for $\varepsilon^{2}$

$$
\begin{aligned}
\frac{\mathrm{d} p_{2}}{\mathrm{~d} \xi} & =\frac{\left[p_{2}\left(h+g-\beta \xi_{\mathrm{i}}\right)-p_{1} \beta \xi_{\mathrm{i}}\left(\xi^{2}-\xi_{\mathrm{i}}^{2}\right)\right]}{\xi_{\mathrm{i}}^{2}\left(\alpha+\beta \xi-2 p_{0}\right)}-\frac{\left[\left(\alpha+\beta \xi-2 p_{0}\right)\left(\xi^{2}-\xi_{\mathrm{i}}^{2}\right)-2 p_{1}\right]\left[p_{1}\left(h+g-\beta \xi_{\mathrm{i}}\right)-p_{0} \beta \xi_{\mathrm{i}}\left(\xi^{2}-\xi_{\mathrm{i}}^{2}\right)\right]}{\xi_{\mathrm{i}}^{2}\left(\alpha+\beta \xi-2 p_{0}\right)^{2}} \\
& +\frac{\left[\left(\alpha+\beta \xi-2 p_{0}\right)\left(\xi^{2}-\xi_{\mathrm{i}}^{2}\right)-2 p_{1}\right]^{2}+\xi\left(\alpha+\beta \xi-2 p_{0}\right)\left[2 p_{1}\left(\xi^{2}-\xi_{\mathrm{i}}^{2}\right)+2 p_{2}\right]\left[p_{0}\left(h+g-\beta \xi_{\mathrm{i}}\right)-h(\alpha+\beta \xi)\right]}{\xi_{\mathrm{i}}^{3}\left(\alpha+\beta \xi-2 p_{0}\right)^{3}} \ldots
\end{aligned}
$$

this case, we define a perturbation parameter $\varepsilon$ as $\varepsilon=1 / \xi_{\mathrm{i}}$. Let us consider the transformation

$\xi=\xi_{\mathrm{i}}\left[1+\varepsilon\left(\xi-\xi_{\mathrm{i}}\right)\right]$

Following the same procedure as that adopted by Hsu and Yang [8], we assume that $p$ can be expanded in terms of $\varepsilon$ as

$p=\sum_{j=0}^{\infty} p_{j} \varepsilon^{j}$
The associated boundary conditions can be obtained by substituting Eq. (16) into Eqs. (14a) and (14b). We obtain

$$
\begin{aligned}
& p_{0}=p^{\mathrm{i}}, \xi=\xi_{\mathrm{i}} \\
& p_{0}=p^{\mathrm{o}}, \xi=\xi_{\mathrm{o}} \\
& p_{s}=0, \xi=\xi_{\mathrm{i}}, s=1,2, \ldots, \\
& p_{s}=0, \xi=\xi_{\mathrm{o}}, s=1,2, \ldots,
\end{aligned}
$$


If $\beta \neq 0$, Eq. (19) can be transformed on the basis of the relation $z=\xi+\alpha / \beta$, and the resultant equation solved subject to Eqs. (22a) and (22b). We obtain

$$
\begin{gathered}
\frac{-1}{\sqrt{M}} \ln \left|\begin{array}{ll}
\frac{\sqrt{M}+\lambda}{\sqrt{M}-\lambda} \sqrt{M}-\lambda^{\mathrm{i}} \\
\quad=\ln +\lambda^{\mathrm{i}}
\end{array}\right| \\
\left(\xi+\frac{\alpha}{\beta}\right) /\left(\xi_{\mathrm{i}}+\frac{\alpha}{\beta}\right) \mid
\end{gathered}
$$

where

$M=\frac{(h+g)^{2}+4 \beta \xi_{\mathrm{i}}\left(h-g+\beta \xi_{\mathrm{i}}\right)}{\xi_{\mathrm{i}}^{2}}$

$\lambda=\frac{4 \beta p_{0}}{\alpha+\beta \xi}+\frac{h+g}{\xi_{\mathrm{i}}}-2 \beta$

$\lambda^{\mathrm{i}}$ is the value of $\lambda$ at $p_{0}=p^{\mathrm{i}}$ and $\xi=\xi_{\mathrm{i}}$.

If $\beta=0$, it can be shown that the solution to Eq. (19) subject to Eqs. (22a) and (22b) is

$$
\begin{array}{r}
\frac{\alpha(g-h)}{(g+h)^{2}} \ln \left|\frac{(g+h) p_{0}-h \alpha}{(g+h) p^{\mathrm{i}}-h \alpha}\right| \\
=\frac{\xi-\xi_{\mathrm{i}}}{\xi_{\mathrm{i}}}+\frac{2}{g+h}\left(p_{0}-p^{\mathrm{i}}\right)
\end{array}
$$

For a highly charged membrane, the effect of coion exclusion implies that $p_{0}$ can be approximated by $(\alpha+\beta \xi)$. In this case, Eq. (20) reduces to

$$
\frac{d p_{1}}{d \xi}+\frac{h-g+\beta \xi_{\mathrm{i}}}{\xi_{\mathrm{i}}(\alpha+\beta \xi)} p_{1}-\frac{2 g\left(\xi-\xi_{\mathrm{i}}\right)}{\xi_{\mathrm{i}}}=0
$$

Solving this equation yields

\section{Results and discussion}

Fig. 2 shows the variation of current efficiency, $\eta$, as a function of the normalized difference between the scaled concentration of fixed charge at the outer liquid-membrane interface and that at the inner membrane-liquid interface, $\left(\rho_{0}-\rho_{\mathrm{i}}\right)=\left(C_{3 \mathrm{o}}-C_{3 \mathrm{i}}\right) / C_{\mathrm{i}}, C_{3 \mathrm{i}}$ and $C_{3 \mathrm{o}}$ being, respectively, the concentrations of fixed charge at the outer liquid-membrane interface and that at the inner membrane-liquid interface for the case $\beta$ in Eq. (13) is positive. Note that, for the present case, $\left(\rho_{\mathrm{o}}-\rho_{\mathrm{i}}\right)=\beta$ which is a measure for the degree of nonuniformity of fixed charge distribution; the smaller its value, the more uniform the distribution of fixed charges. If $\left(\rho_{\mathrm{o}}-\rho_{\mathrm{i}}\right)$ vanishes, the fixed charges are uniformly distributed. Fig. 2 reveals that if $\left(\rho_{\mathrm{o}}-\rho_{\mathrm{i}}\right)$ vanishes, $\eta$ is independent of $\xi_{\mathrm{i}}$. For a fixed $\left(\rho_{\mathrm{o}}-\rho_{\mathrm{i}}\right), \eta$ increases with $\xi_{i}$. This implies that the smaller the curvature of a membrane, the greater the current efficiency. Note that, if $\xi_{\mathrm{i}}$ is sufficiently large, $\eta$ increases with $\left(\rho_{\mathrm{o}}-\rho_{\mathrm{i}}\right)$; the reverse is true if it is small. In other words, the current efficiency of a membrane with a nonuniform fixed charge distribution may not be higher than that with a uniform fixed charge distribution. The corresponding results for the case where $\beta$ is negative are shown in Fig. 3. As can be seen from this figure, the trends illustrated in Fig. 2 can be reversed by changing the sign of $\beta$. Therefore, the current efficiency of a membrane can be raised by choosing its parameters appropriately. Note that, since the problem under consideration is of nonlinear

$$
\begin{aligned}
p_{1}= & \frac{\left(\beta^{2} G+\beta C G\right) \xi^{2}+\left(\alpha C G-2 G \xi_{\mathrm{i}} \beta^{2}-\beta C G \xi_{\mathrm{i}}\right) \xi-\left(\alpha^{2} G+\alpha C G \xi_{\mathrm{i}}+2 \alpha \beta G \xi_{\mathrm{i}}\right)}{(C+\beta)(C+2 \beta)} \\
& +\frac{(\alpha+\beta \xi)^{-C / \beta}\left(2 \beta^{2} L+3 \beta L C+L C^{2}\right)}{(C+\beta)(C+2 \beta)\left(2 \beta^{2} K+3 \beta K C+K C^{2}\right)}
\end{aligned}
$$

where $\quad C=\left(h-g+\beta \xi_{\mathrm{i}}\right) / \xi_{\mathrm{i}}, \quad G=2 g / \xi_{\mathrm{i}}$, $K=(\alpha+\beta e)^{-C / \beta}$, and $L=\beta^{2} \mathrm{Ge}^{2}+2 \alpha \beta \mathrm{Ge}+\alpha^{2} \mathrm{G}$. Substituting $p_{\mathrm{j}}$ into Eq. (16) gives the perturbation solution of Eq. (13). If $\varepsilon$ vanishes (or $\xi_{\mathrm{i}}$ approaches infinity), the curvature of the spherical membrane vanishes, and it can be treated as a planar membrane. In other words, the zeroth-order solution of Eq. (13), $p_{0}$, is the exact solution for the case of a planar membrane. nature, as suggested by Eq. (13), we have $\left[\eta\left(\rho_{\mathrm{o}}-\rho_{\mathrm{i}}\right)-\eta(0)\right] \neq\left[\eta(0)-\eta\left(\rho_{\mathrm{i}}-\rho_{\mathrm{o}}\right)\right] . \quad$ Figs. 2 and 3 suggest that, if fixed charge is uniformly distributed, the current efficiency is independent of the inner radius and the current density.

The variation of current efficiency, $\eta$, as a function of $\left(\rho_{\mathrm{o}}-\rho_{\mathrm{i}}\right)$ for various scaled current density $i$ is shown in Fig. 4 for the case where the slope $\beta$ in Eq. (10) is positive. This figure suggests that, if the 


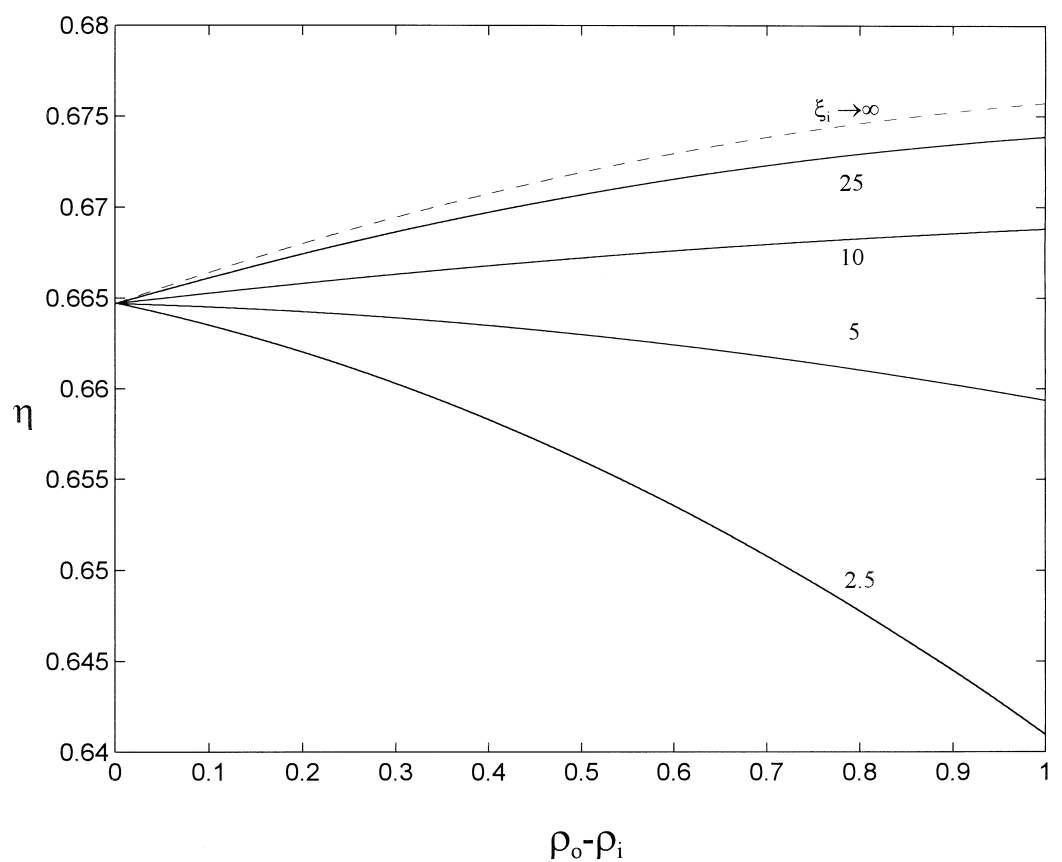

Fig. 2. Variation of current efficiency $\eta$ as a function of $\left(\rho_{\mathrm{o}}-\rho_{\mathrm{i}}\right)$ at various scaled inner radius of a membrane $\xi_{\mathrm{i}}$ for the case of positive $\beta$, $i=1$, and $\gamma=1$.

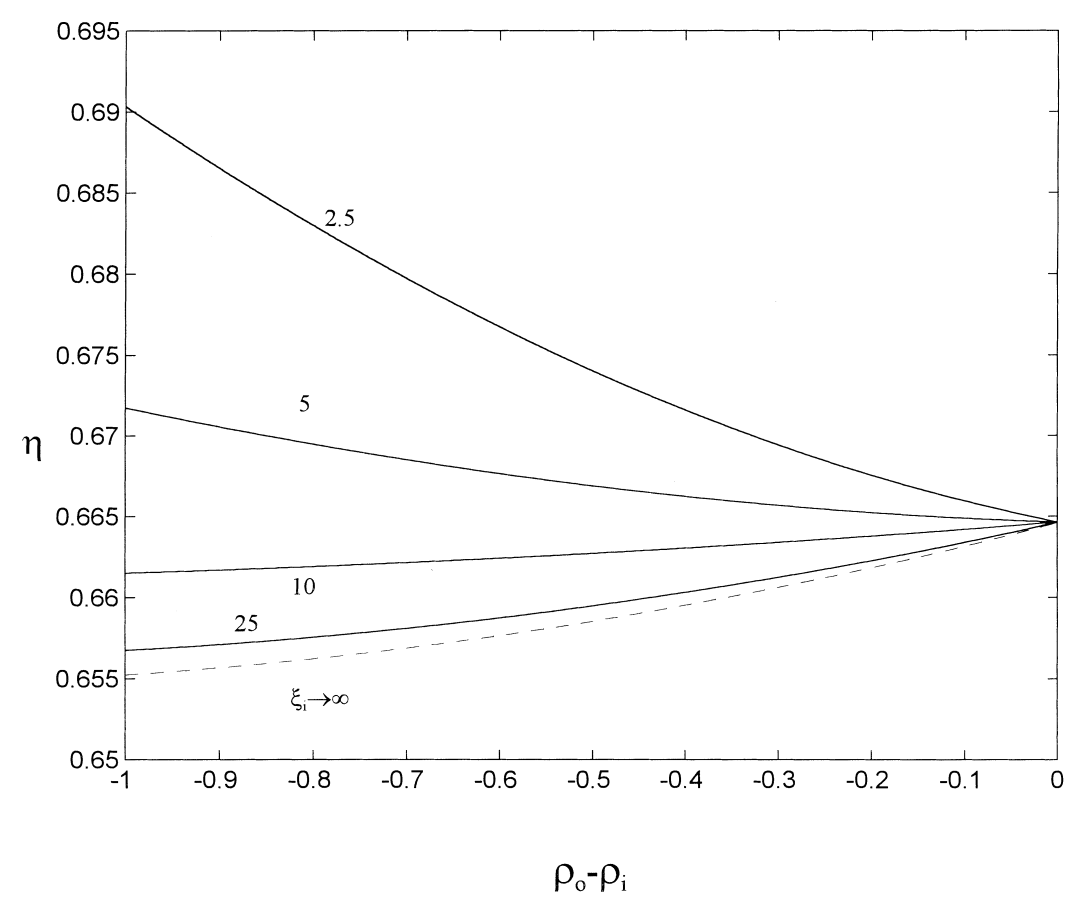

Fig. 3. Variation of current efficiency $\eta$ as a function of $\left(\rho_{\mathrm{o}}-\rho_{\mathrm{i}}\right)$ at various scaled inner radius of a membrane $\xi_{\mathrm{i}}$ for the case of negative $\beta$, $i=1$, and $\gamma=1$. 


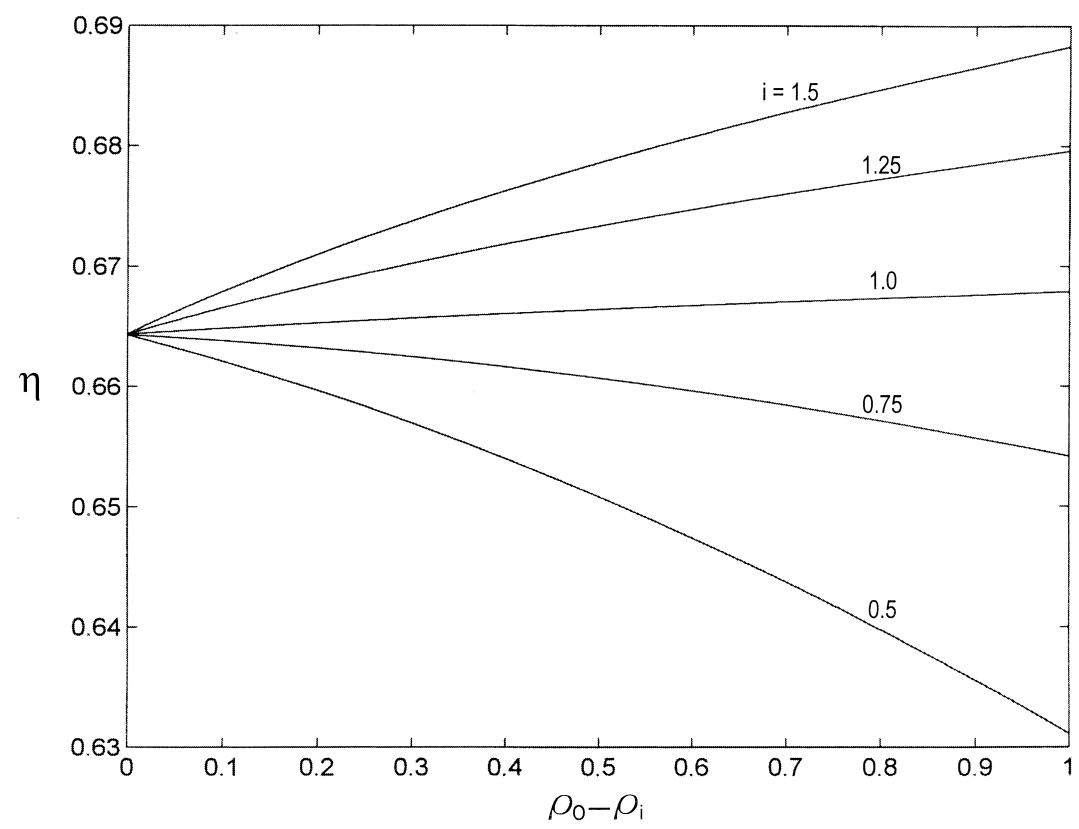

Fig. 4. Variation of current efficiency $\eta$ as a function of $\left(\rho_{\mathrm{o}}-\rho_{\mathrm{i}}\right)$ at various scaled current density $i$ for the case of positive $\beta$, $\xi_{\mathrm{i}}=10$, and $\gamma=1$.

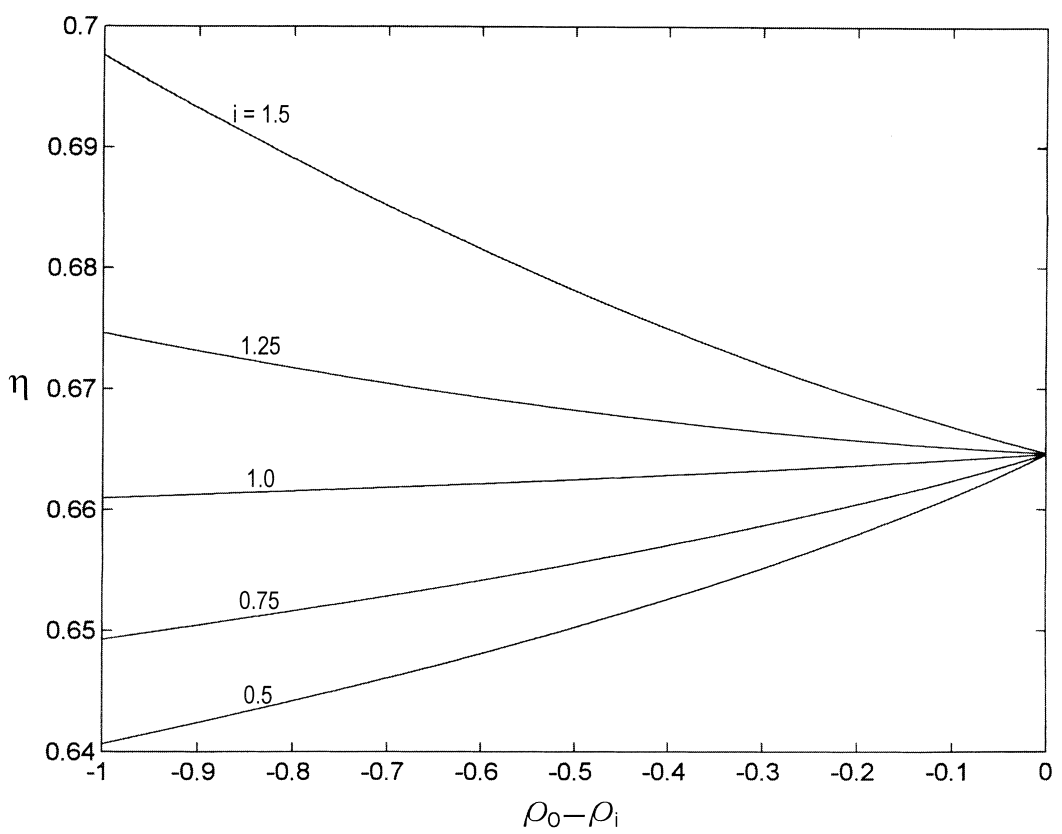

Fig. 5. Variation of current efficiency $\eta$ as a function of $\left(\rho_{\mathrm{o}}-\rho_{\mathrm{i}}\right)$ at various scaled current density $i$ for the case of negative $\beta$, $\xi_{\mathrm{i}}=10$, and $\gamma=1$. 


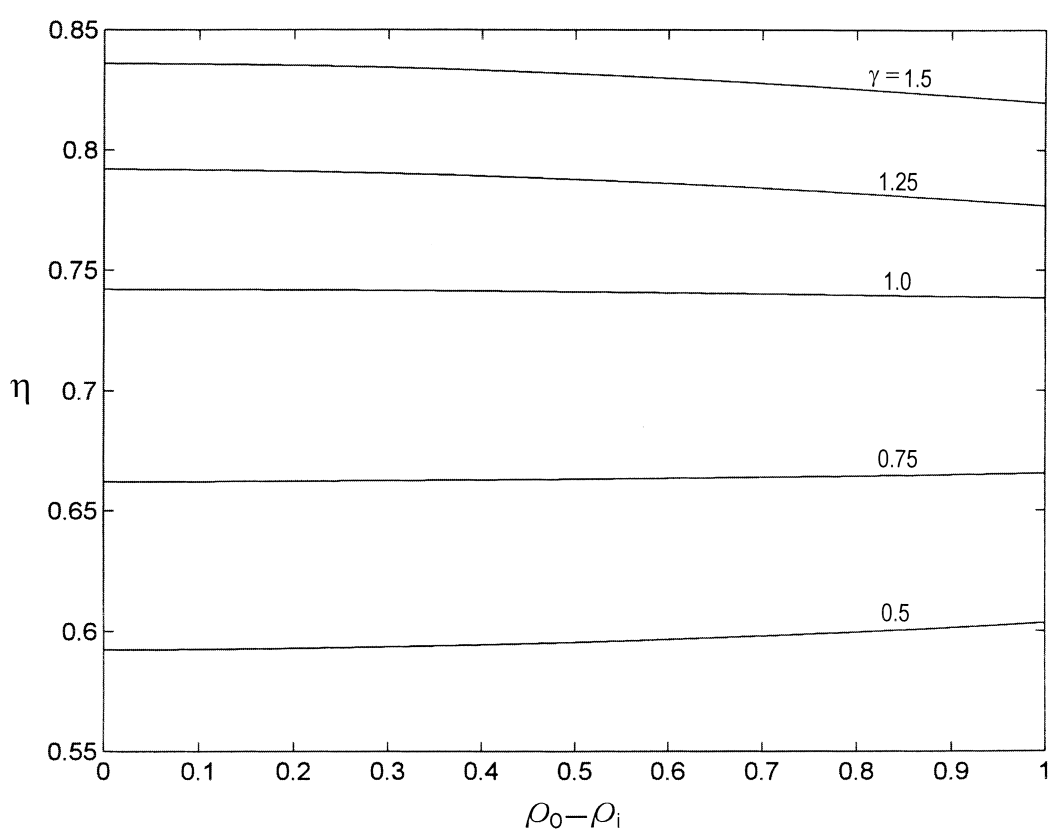

Fig. 6. Variation of current efficiency $\eta$ as a function of $\left(\rho_{\mathrm{o}}-\rho_{\mathrm{i}}\right)$ at various scaled mean fixed charge concentration $\gamma$ for the case of positive $\beta, \xi_{\mathrm{i}}=10$, and $i=1$.

fixed charge in the membrane phase is uniformly distributed, i.e. $\left(\rho_{\mathrm{o}}-\rho_{\mathrm{i}}\right)=0, \eta$ is independent of $i$. For a fixed $\left(\rho_{\mathrm{o}}-\rho_{\mathrm{i}}\right), \eta$ increases with $i$. If $i$ is sufficiently high, $\eta$ increases with $\left(\rho_{\mathrm{o}}-\rho_{\mathrm{i}}\right)$, and the reverse is true, if it is low. The corresponding results for the case where $\beta$ is negative are presented in Fig. 5. Again, this figure illustrates that $\eta$ can be raised by varying the sign of $\beta$, that is increasing the degree of nonuniformity of fixed charge distribution.

Fig. 6 shows the variation of current efficiency $\eta$ as a function of $\left(\rho_{\mathrm{o}}-\rho_{\mathrm{i}}\right)$ for various mean fixed charge concentration $\gamma$ for the case where the slope $\beta$ in Eq. (10) is positive. The corresponding results for the case where $\beta$ is negative are shown in Fig. 7. Fig. 6 reveals that, for a fixed $\left(\rho_{\mathrm{o}}-\rho_{\mathrm{i}}\right), \eta$ increases with $\gamma$. If $\gamma$ is small, $\eta$ increases with $\left(\rho_{\mathrm{o}}-\rho_{\mathrm{i}}\right)$, and the reverse is true if $\gamma$ is large. Figs. 6 and 7 reveal that, regardless of the sign of $\beta, \eta$ increases with $\gamma$. Also if $\gamma$ is close to unity, $\eta$ is insensitive to the variation in $\left(\rho_{\mathrm{o}}-\rho_{\mathrm{i}}\right)$.

The qualitative behavior of a spherical membrane illustrated in Figs. 2-7 is similar to that of a cylindrical membrane [8]. Also, as pointed out by Sokirko et al. [11] and Hsu et al. [12], the effect of the inhomo- geneity of fixed charge distribution on the current efficiency is less significant than that of the boundary layers near the liquid-membrane interfaces. The former is on the order of 5\%, and the latter can exceed $10 \%$.

As in the case of a cylindrical membrane [8], when the effect of curvature on current efficiency is examined, the width of a membrane and its volume cannot be fixed simultaneously. Similarly, the average fixed charge concentration and the total amount of fixed charges cannot be fixed at the same time. In the numerical simulation, the width of a membrane and the average fixed charge concentration are assumed to be constant.

It is found that the assumption of coion exclusion is satisfied, in general, if a membrane is highly charged. Table 1 illustrates the deviation arising from the assumption of coion exclusion. This table reveals that, if the concentration of fixed charge is of the same order as that of bulk liquid concentration, the deviation in current efficiency can be appreciable. On the other hand, if the concentration of fixed charge is high, the deviation becomes negligible. 


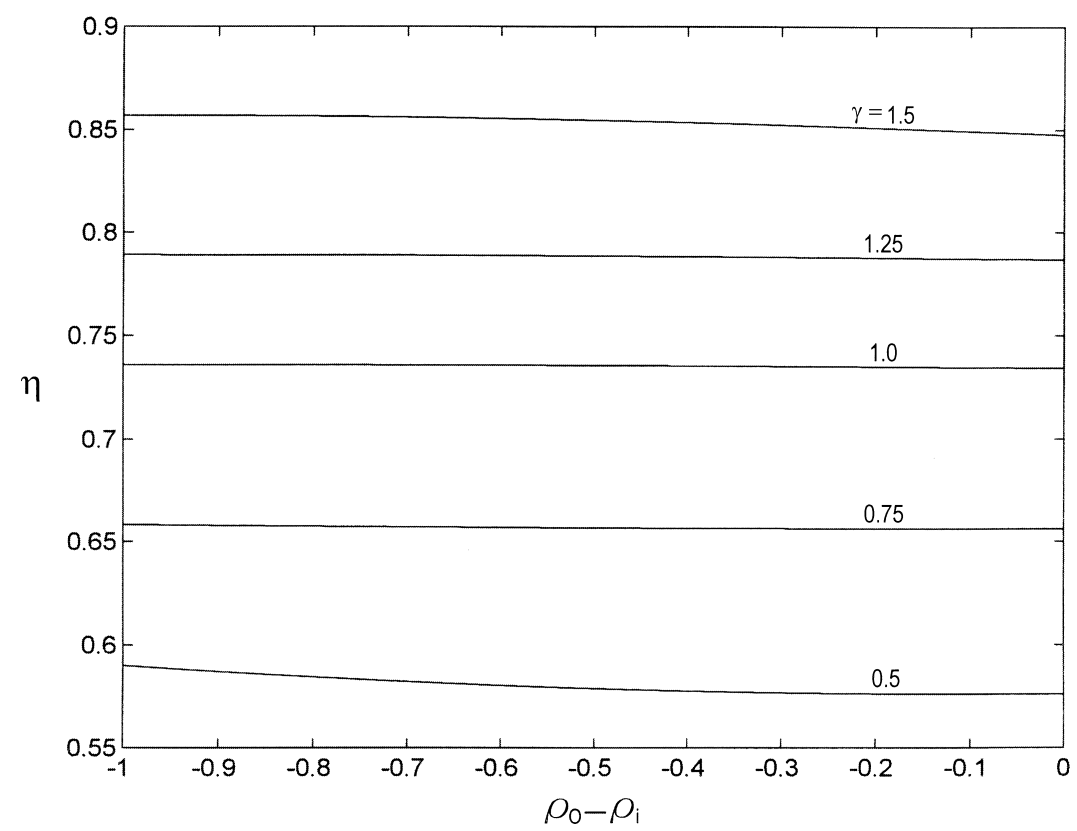

Fig. 7. Variation of current efficiency $\eta$ as a function of $\left(\rho_{\mathrm{o}}-\rho_{\mathrm{i}}\right)$ at various scaled mean fixed charge concentration $\gamma$ for the case of negative $\beta, \xi_{\mathrm{i}}=10$, and $i=1$.

\subsection{Planar and cylindrical membranes}

Eq. (1) can be generalized to include planar, cylindrical, and spherical membranes as

$\frac{J_{j}}{r^{m}}=-D_{j}\left[\frac{\mathrm{d} C_{j}}{\mathrm{~d}_{r}}+(-1)^{j+1} \frac{q}{k_{\mathrm{B}} T} \frac{\mathrm{d} \phi}{\mathrm{d} r} C_{j}\right], j=1,2$

where $m$ is a shape index, $m=0,1$, and 2 for planar,

Table 1

Deviation in the current efficiency estimated on the basis of the coion-exclusion assumption from the corresponding exact value based on Eq. (13) for the case of a spherical membrane with $i=0.5, \xi_{\mathrm{i}}=5$, and $\left(\rho_{\mathrm{o}}-\rho_{\mathrm{i}}\right)=0.5$

\begin{tabular}{llll}
\hline$\gamma^{\text {a }}$ & Exact $\eta$ & Approximate $\eta$ & Deviation (\%) \\
\hline 1 & 0.664 & 0.609 & 8.352 \\
2 & 0.798 & 0.758 & 4.912 \\
3 & 0.859 & 0.834 & 2.867 \\
4 & 0.893 & 0.876 & 1.931 \\
5 & 0.912 & 0.899 & 1.368 \\
\hline
\end{tabular}

${ }^{\mathrm{a}} \gamma$ is the mean fixed charge concentration. $\gamma=1$ corresponds to the case that the mean fixed charge concentration is the same as the bulk concentration of ions. cylindrical, and spherical membranes, respectively. Here, $r_{\mathrm{o}}$ and $r_{\mathrm{i}}$ are the outer and inner radii of a cylindrical membrane, respectively. The origin of the coordinate is located at the left liquid-membrane interface for a planar membrane, and at the axis of a cylindrical membrane. $C_{\mathrm{i}}$ and $C_{\mathrm{o}}$ are the bulk concentrations of ions inside and outside a cylindrical membrane, and those on the left and right sides of a planar membrane, respectively.

The Poisson equation needs to be modified as

$\frac{\mathrm{d}^{2} \phi}{\mathrm{d} r^{2}}+\frac{m}{r} \frac{\mathrm{d} \phi}{\mathrm{d} r}=-\frac{4 \pi q}{\omega}\left(C_{1}-C_{2}-C_{3}\right)$

Also, Eqs. (7a), (7b) and (7d) become, respectively,

$h=\frac{J_{1}}{D C_{\mathrm{i}} r_{\mathrm{i}} W^{m-2}}$
$g=\frac{J_{2}}{D C_{\mathrm{i}} r_{\mathrm{i}} W^{m-2}}$

and

$i=h_{c}-g_{c}=\frac{I}{D C_{\mathrm{i}} F W^{m-1}}$

For a planar membrane, the scaled mean fixed 
charge concentration is defined as

$\gamma=\int_{0}^{1}(\alpha+\beta \xi) \mathrm{d} \xi=\alpha+\frac{\beta}{2}$

Also, Eq. (13) reduces to

$\frac{\mathrm{d} p}{\mathrm{~d} \xi}=\frac{p\left(h_{c}+g_{c}-\beta\right)-h_{c}(\alpha+\beta \xi)}{(\alpha+\beta \xi-2 p)}$

This is consistent with the result derived by Hsu and Ting [4] and Hsu et al. [12].

For a cylindrical membrane, the scaled mean fixed charge concentration is defined as

$$
\begin{aligned}
\gamma & =\left[2 \pi \int_{\xi_{\mathrm{i}}}^{\xi_{\mathrm{o}}}(\alpha+\beta \xi) \xi \mathrm{d} \xi\right] /\left[\pi\left(\xi_{\mathrm{o}}^{2}-\xi_{\mathrm{i}}^{2}\right)\right] \\
& =\left[\frac{\alpha}{2}\left(\xi_{\mathrm{o}}^{2}-\xi_{\mathrm{i}}^{2}\right)+\frac{\beta}{3}\left(\xi_{\mathrm{o}}^{3}-\xi_{\mathrm{i}}^{3}\right)\right] /\left[\frac{1}{2}\left(\xi_{\mathrm{o}}^{2}-\xi_{\mathrm{i}}^{2}\right)\right]
\end{aligned}
$$

Eq. (13) becomes

$\frac{\mathrm{d} p}{d \xi}=\frac{p\left(h_{c}+g_{c}-\beta \xi\right)-h_{c}(\alpha+\beta \xi)}{\xi(\alpha+\beta \xi-2 p)}$

This is consistent with the result of Hsu and Yang [8].

\section{Conclusion}

A general expression for the transport of ions in a planar, cylindrical, and spherical ion-selective membrane is discussed. A perturbation method is adopted to solve the governing equation for the case of linear fixed charge distribution in the membrane phase. We show that if the fixed charge is uniformly distributed, the current efficiency is independent of both, the curvature (or inner radius) and current density. For a fixed total amount of fixed charges, the current efficiency increases with the degree of nonuniformity of fixed charge distribution.

\section{List of symbols}

$C_{\mathrm{i}} \quad$ bulk concentration of ions inside membrane $\left(\mathrm{mol} / \mathrm{m}^{3}\right)$

$C_{\mathrm{o}} \quad$ bulk concentration of ions outside membrane $\left(\mathrm{mol} / \mathrm{m}^{3}\right)$
$C_{1} \quad$ concentration of cations $\left(\mathrm{mol} / \mathrm{m}^{3}\right)$

$C_{2}$ concentration of anions $\left(\mathrm{mol} / \mathrm{m}^{3}\right)$

$C_{3}$ concentration of fixed charge $\left(\mathrm{mol} / \mathrm{m}^{3}\right)$

$C_{3 \mathrm{i}} \quad$ value of $C_{3}$ at $r_{\mathrm{i}}\left(\mathrm{mol} / \mathrm{m}^{3}\right)$

$C_{30} \quad$ value of $C_{3}$ at $r_{\mathrm{o}}\left(\mathrm{mol} / \mathrm{m}^{3}\right)$

$D$ diffusivity of ion $\left(\mathrm{m}^{2} / \mathrm{s}\right)$

$D_{1} \quad$ diffusivity of cation $\left(\mathrm{m}^{2} / \mathrm{s}\right)$

$D_{2}$ diffusivity of anion $\left(\mathrm{m}^{2} / \mathrm{s}\right)$

$F \quad$ Faraday constant (Coulomb/mol)

$g \quad$ scaled rate of transfer of cation

$g_{\mathrm{c}} \quad=g r_{\mathrm{i}} / \mathrm{W}$

$h$ scaled rate of transfer of anion

$h_{\mathrm{c}} \quad=h r_{\mathrm{i}} / \mathrm{W}$

$i \quad$ scaled current

I current (Coulomb/s)

$J_{1} \quad$ flux of cation $\left(\mathrm{mol} / \mathrm{m}^{2} / \mathrm{s}\right)$

$J_{2}$ flux of anion $\left(\mathrm{mol} / \mathrm{m}^{2} / \mathrm{s}\right)$

$k_{\mathrm{B}} \quad$ Boltzmann constant $(\mathrm{J} / \mathrm{K})$

$m$ shape index

$M \quad$ parameter defined in Eq. (23a)

$n \quad$ scaled concentration of anion

$p \quad$ scaled concentration of cation

$p_{j} \quad$ perturbation function defined in Eq. (16)

$p^{\mathrm{i}} \quad$ value of $p$ at $\xi=\xi_{\mathrm{i}}$

$p^{\text {o }} \quad$ value of $p$ at $\xi=\xi_{\text {o }}$

$q$ elementary charge (Coulomb)

$r \quad$ radial distance $(\mathrm{m})$

$r_{\mathrm{i}} \quad$ inner radius of membrane $(\mathrm{m})$

$r_{\mathrm{o}} \quad$ outer radius of membrane $(\mathrm{m})$

$T$ temperature (K)

$W \quad$ width of membrane (m)

\section{Greek letters}

$z=\xi+\alpha / \beta$

$\alpha \quad$ parameter defined in Eq. (10)

$\beta \quad$ parameter defined in Eq. (10)

$\gamma \quad$ scaled mean fixed charge concentration

$\varepsilon \quad$ perturbation parameter

$\eta \quad$ current efficiency

$\theta \quad=C_{\mathrm{o}} / C_{\mathrm{i}}$

$\lambda \quad$ parameter defined in Eq. (23b)

$\xi \quad$ scaled radial distance

$\xi_{\mathrm{i}} \quad$ scaled inner radius

$\xi_{\mathrm{o}} \quad$ scaled outer radius

$\rho$ scaled concentration of fixed charge

$\rho_{\mathrm{i}} \quad=C_{3 \mathrm{i}} / C_{\mathrm{i}}$ 
$\rho_{\mathrm{o}} \quad=C_{3 \mathrm{o}} / C_{\mathrm{i}}$

$\phi \quad$ electrical potential (V)

$\psi \quad$ scaled electrical potential

$\omega$ dielectric constant (Coulomb $\left.{ }^{2} / \mathrm{J} / \mathrm{m}\right)$

\section{Acknowledgements}

The National Science Council of the Republic of China partially financially supports this work.

\section{References}

[1] H. Reiss, I.C. Bassignana, Critique of the mechanism of superselectivity in ion exchange membranes, J. Membr. Sci. 11 (1982) 219.

[2] J. A Manzanares, S. Mafe, J. Pellicer, Transport phenomena and asymmetry effects in membranes with asymmetric fixed charge distributions, J. Phys. Chem. 95 (1991) 5620.

[3] J. Manzanares, S. Mafe, J. Pellicer, Current efficiency enhancement in membranes with macroscopic inhomogeneities in the fixed charge distribution, J. Chem. Soc. Faraday Trans. 88 (1992) 2355.
[4] J.P. Hsu, K.C. Ting, Current efficiency of an ion-selective membranes: effect of fixed charge distribution, J. Electrochem. Soc. 145 (1998) 1088.

[5] R.P. Buck, Kinetics of bulk and interfacial ionic motion: microscopic bases and limits for the Nernst-Planck equation applied to membrane systems, J. Membr. Sci. 17 (1984) 1.

[6] J.P. Hsu, B.T. Liu, Current efficiency of ion-selective membranes: effects of local electroneutrality and Donnan equilibrium, J. Phys. Chem. 101 (1997) 7928.

[7] I. Rubinstein, B. Zaltzman, O. Kedem, Electric fields in and around ion-exchange membranes, J. Membr. Sci. 125 (1997) 17.

[8] J.P. Hsu, K.L. Yang, Transport of ions through cylindrical ionselective membranes, J. Phys. Chem. 100 (1996) 12503.

[9] J.P. Hsu, B.T. Liu, Transport of ions through elliptic ionselective membranes, J. Membr. Sci. 142 (1998) 245.

[10] R.J. Hunter, Foundations of Colloid Science, Oxford University Press, London, vol. 1, 1989.

[11] V.A. Sokirko, J.A. Manzanares, J. Pellicer, The permselectivity of membrane systems with an inhomogeneous distribution of fixed charge groups, J. Coll. Interface Sci. 168 (1994) 32.

[12] J.P. Hsu, K.L. Yang, K.C. Ting, Effect of convective boundary layer on the current efficiency of a membrane bearing nonuniformly distributed fixed charges, J. Phys. Chem. 101 (1997) 8984. 